\title{
Intermittently-Powered Energy Harvesting Step Counter for Fitness Tracking
}

\author{
Alberto Rodriguez ${ }^{\dagger}$, Domenico Balsamo ${ }^{\dagger}$, Zhenhua Luo $^{\ddagger}$, Steve P. Beeby ${ }^{\dagger}$, Geoff V. Merrett ${ }^{\dagger}$, Alex S. Weddell ${ }^{\dagger}$ \\ ${ }^{\dagger}$ Department of Electronics and Computer Science, University of Southampton \\ ${ }^{\ddagger}$ School of Water, Energy and Environment, Cranfield University \\ Email: ${ }^{\dagger}\{$ ara1g13,db2a12,spb,gvm,asw $\} @$ ecs.soton.ac.uk, ${ }^{\ddagger}$ z.luo@ $@$ cranfield.ac.uk
}

\begin{abstract}
Over the past decade, there has been a rapid increase in the popularity of wearable and portable devices, such as step counters, to monitor fitness performance. However, these devices are battery-powered, meaning that their lifetimes are restricted by battery capacity. Ideally, wearable devices could be powered by energy harvested from human motion. Energy harvesting systems traditionally incorporate energy storage to cope with source variability. However, energy storage takes time to charge and increases the size and cost of systems. This paper proposes an intermittently-powered energy harvesting step counter for integrated wearable applications, which aims to remove the energy storage element. The proposed step counter sustains its operation by harvesting energy from footsteps using a ferroelectret insole, which also works as an event detection sensor, i.e. the system is powered by the parameter that is being sensed. Designing this required the characterization of the insole to evaluate the amount of energy provided, and analysis of the energy needed by the overall system. Finally, the system was implemented and experimentally validated. The proposed step counter has an error of less than $4 \%$ when walking, which is lower than the error in conventional smartphone applications.

Index Terms-Step Counter, Energy Harvesting, Event Sensor, Ferroelectret Insole, Intermittent Source.
\end{abstract}

\section{INTRODUCTION}

Typical systems to monitor fitness metrics such as number of steps, distance walked, calorie consumption, etc. are battery-powered, meaning that their lifetime becomes restricted by battery capacity and discharge rate [1], [2]. For example, smart watches for fitness performance tracking typically last for 18-20 hours, depending on the model and activity [3], while fitness trackers can last for 3-4 days [4], having an average charge time of 2 hours.

Motivated by the limited lifespan achievable with batterypowered systems, research has recently looked at replacing batteries with energy harvesting solutions. For wearable systems, the energy harvested from human motion (e.g. footsteps) is attractive, potentially avoiding periodic battery replacement or charging [5]. However, the power obtained from human motion is typically in the range of $\mathrm{mW}$, intermittent and of short duration [6], [7]. To sustain computation and mitigate variability, energy harvesting systems normally integrate energy storage units, which require time to charge and increase their volume, mass and cost.

This paper proposes an intermittently-powered energy harvesting step counter for integrated wearable applications. In contrast to conventional energy harvesting systems, the pro-

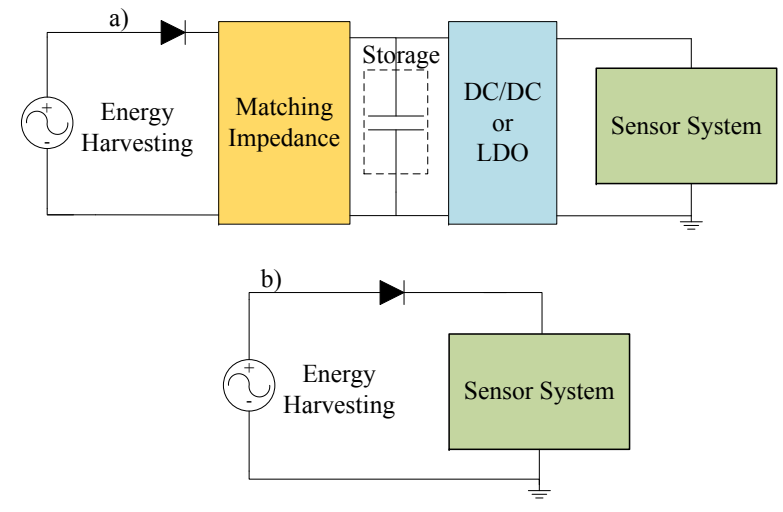

Fig. 1. Energy harvesting systems. a) Conventional system with energy storage. b) Storage-less energy harvesting system.

posed step counter removes the energy storage element and operates directly from the harvesting source (Figure 1). It sustains its operation by harvesting energy from footsteps, using a ferroelectret insole which generates electricity under mechanical stress [8]. The system uses a microcontroller (MCU), with a low-power non-volatile memory, which wakes up at each step and retains data during the power outage between steps. The ferroelectret insole also acts as an event detection sensor: the system is only powered when the event (i.e. a footstep) is detected.

The novel contributions of this work are:

- Characterization of the dynamics of human powered energy harvesting, with a ferroelectret insole (Section II).

- Analysis of the energy requirements and a methodology for designing the step counter (Section III).

- Design and practical testing of a self-powered step counter. (Section IV).

Experiments demonstrate that the proposed step counter has an error in counting steps of less than $4 \%$ when walking, significantly better than existing smartphone applications.

\section{Ferroelectret Insole Characterization}

In this section, we look at the performance of the ferroelectret insole when mounted in a shoe. We also calculate the energy generated per step, which aids the design of the system in Section III.

Ferroelectret materials are flexible cellular polymer foams that, similarly to a piezoelectric source, convert mechanical 


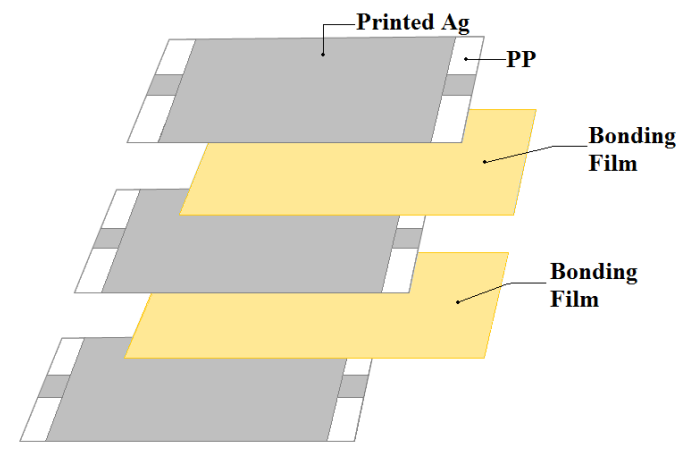

Fig. 2. Structure of a multilayer ferroelectret insole.

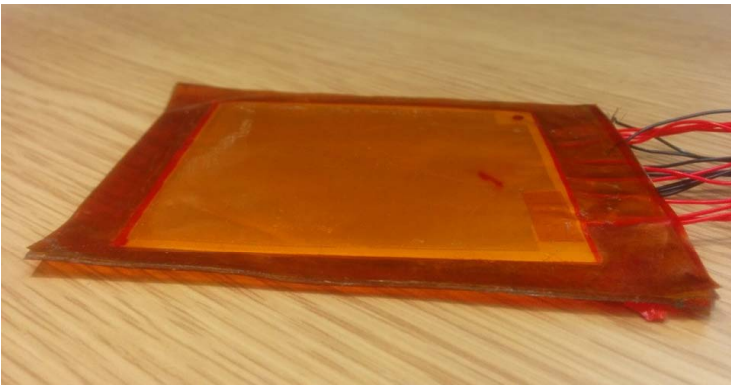

Fig. 3. 30-layer ferroelectret insole used in our design.

energy into electricity when they are bent or compressed [9]. In an energy harvesting insole made with ferroelectret materials, the mechanical stress is generated by the heel striking against the floor when walking or running. Figure 2 shows the model of a three-layer insole of polypropylene ferroelectret (PP) connected in parallel with bonding films between layers [10]. A similar insole with 30 layers has been used in our design (Figure 3).

As shown in Figure 4, these harvesting sources generate high-power energy bursts for a short period of time (in the order of $\mathrm{ms}$ ). The magnitude of these power bursts depends on the momentum of applied compression and the impedance of the load that is connected to the source. The greater the momentum, the greater the voltage but the shorter the duration of the power burst. The ferroelectret insole generates two voltage peaks at each step: positive when compressed and negative when released.

Although this work aims to negate the need for large energy buffers, some capacitance is inherently required by microcontrollers to decouple the energy source (and possible electrical noise) from the system. This is known as decoupling capacitance. In intermittently-powered systems [11], [12], this capacitance may also be exploited as a small energy store. In order to evaluate the performance of the ferroelectret insole when connected to a microcontroller, we use a range of capacitance values. The values chosen ranged between the minimum decoupling capacitance recommended by manufacturer for a typical MCU (i.e. $4.7 \mu \mathrm{F}$ ), and the value incorporated in a reference design for that device (i.e. $16 \mu \mathrm{F}$ ) [13].

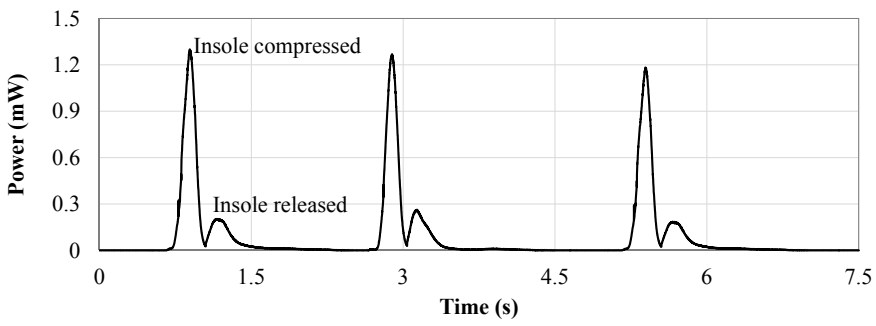

Fig. 4. Rectified power signal generated by the ferroelectret insole with a high-impedance workload after three steps.

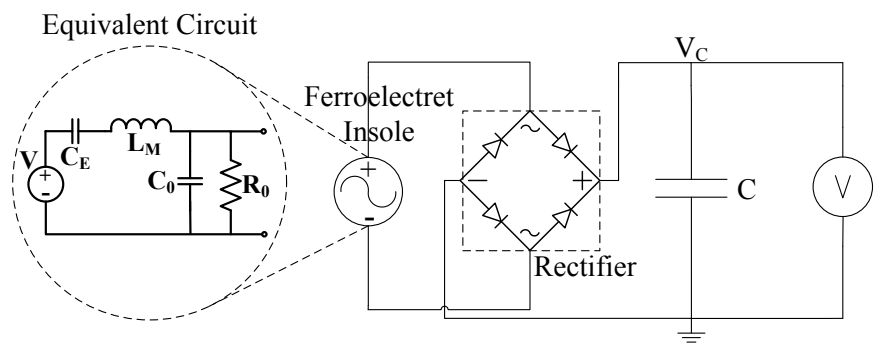

Fig. 5. Characterization circuit including the full-wave rectifier.

Figure 5 shows the characterization circuit with a purely capacitive load. The circuit includes a bridge-rectifier connected at the insole's output. The ferroelectret insole is represented by a basic equivalent circuit for piezoelectric transducers [14]. The capacitance $C_{E}$ is the inverse of the mechanical elasticity of the insole. $L_{M}$ represents the seismic mass of the transducer. The capacitor $C_{0}$ is the static capacitance of the harvesting source and $R_{0}$ is the resistance of the dielectric material that forms the static capacitance (insulation resistance), whose ideal value should be over $10^{12} \Omega$.

The voltage $\left(V_{C}\right)$ is measured across the capacitor, and the electrical energy generated at each step is calculated. The experiment was performed by a 70-kg person moving at two different step rates (referred to herein as operating modes): walking (approximately 70 steps per minute) and running (approximately 120 steps per minute). This test was executed five times for each capacitor in each mode and the results were averaged.

Figure 6 shows the voltage measured with each capacitor after each step in running and walking modes. Here, $V_{\min }$ is the minimum operating voltage of the typical low-power MCU. This allows us to observe when an MCU would became active. In running mode, the force applied to the insole is higher than in walking mode, and produces a higher voltage increment at each step. This introduces uncertainty related to the number of steps walked before the system becomes active. This uncertainty increases with larger values of decoupling capacitance. For example, with the lowest capacitance, $V_{\min }$ is reached after 2 or 3 steps in both walking modes, while with higher capacitances the voltage threshold is not reached even after 5 steps.

The energy generated per footstep can also be calculated from this test by using the following equation: 


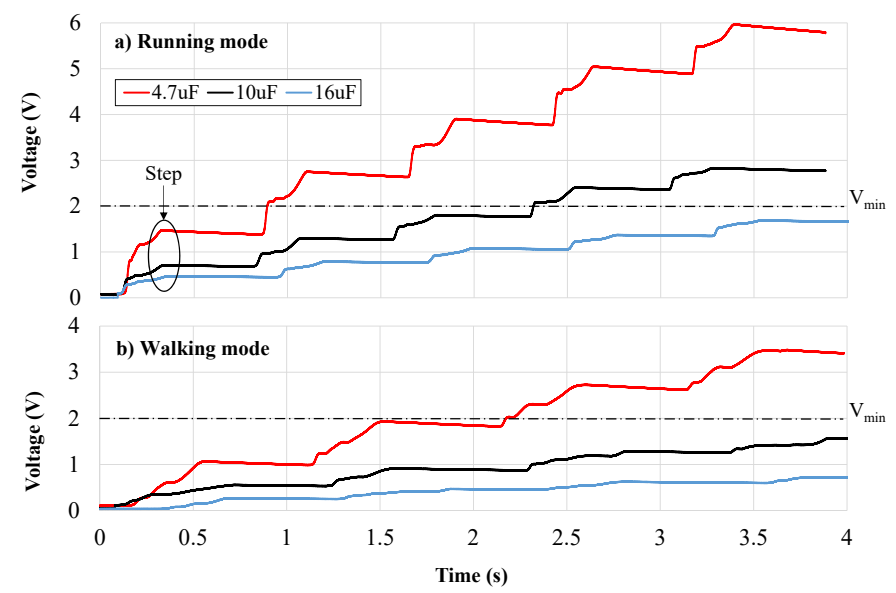

Fig. 6. Ferroelectret insole characterization with three different values of capacitances in walking and running mode.

TABLE I

MEAN ENERGy OBTAINED PER SINGLE STEP

\begin{tabular}{|c|c|c|c|c|c|c|c|c|}
\hline \multirow{2}{*}{$\begin{array}{c}\text { Capacitance } \\
(\mu \mathrm{F})\end{array}$} & \multicolumn{4}{|c|}{ Walking mode $(\mu \mathrm{J})$} & \multicolumn{4}{c|}{ Running mode $(\mu \mathrm{J})$} \\
\cline { 2 - 9 } & $1^{\text {st. }}$ Step & $2^{\text {nd. }}$ Step & $3^{\text {rd. }}$ Step & Total & $1^{\text {st. }}$ Step & $2^{\text {nd. }}$ Step & $3^{\text {rd. }}$ Step & Total \\
\hline 4.7 & 2.35 & 7.05 & 9.21 & 18.61 & 5.30 & 13.14 & 18.89 & 37.33 \\
\hline 10 & 0.85 & 1.27 & 1.41 & 3.53 & 3.21 & 2.47 & 4.66 & 10.34 \\
\hline 16 & 0.21 & 0.37 & 0.73 & 1.31 & 0.59 & 0.92 & 1.33 & 2.84 \\
\hline
\end{tabular}

$$
E_{\text {capacitor }}=C \frac{V_{2}^{2}-V_{1}^{2}}{2}
$$

where $C$ is the decoupling capacitance, $V_{1}$ is the voltage in the capacitor before the step and $V_{2}$ is the voltage reached after the footstep is taken.

Table I shows the average energy that the ferroelectret insole charges into each capacitor in each mode. The maximum amount of energy is obtained with the lowest capacitance, being up to fourteen times higher. Therefore, with the capacitance of $4.7 \mu \mathrm{F}$ the insole gives more energy per footstep and allows the MCU to become active more quickly.

\section{OVERALl SYSTEM DESIGN}

The insole characterization performed in Section II did not consider the effect of an active load, which causes a fast discharge of the decoupling capacitance and a consequent voltage drop across the MCU. Describing this requires the knowledge of the overall system, the energy requirements when operating in different modes (i.e. start-up, active and low-power) and the usage of the energy buffered during these modes to efficiently complete a task (i.e. count a step).

\section{A. System Architecture}

Figure 7 represents a simplified example of the system architecture, where the ferroelectret insole output is rectified and used to power the sensor system, which is composed of an evaluation MSP-EXP430FR5739 test board [13]. This board contains an MCU with a non-volatile Ferroelectric RAM (FRAM) memory and a $4.7 \mu \mathrm{F}$ decoupling capacitance $C$ from

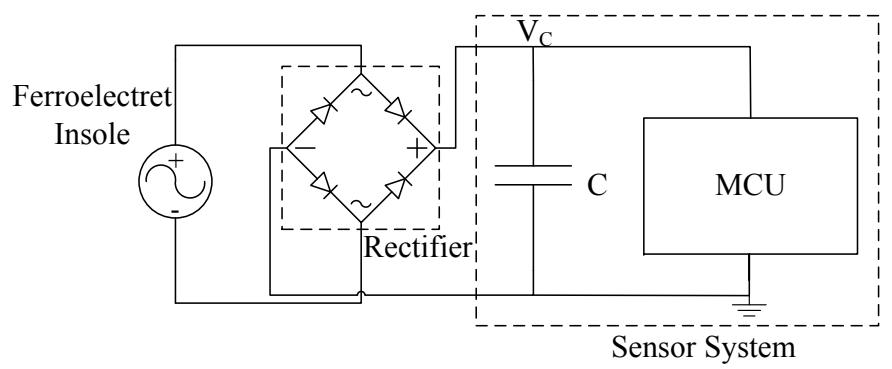

Fig. 7. Simplified system architecture of the step counter with ferroelectret insole.

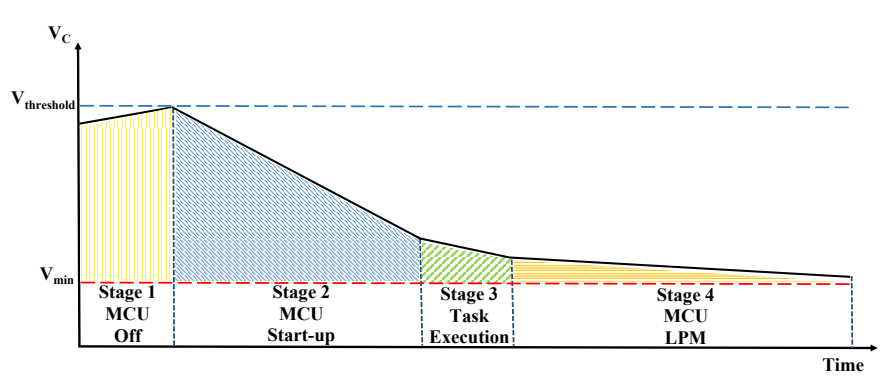

Fig. 8. Stages in which is divided the process of counting a step.

the insole characterization (see Section II). This board also integrates a 3-axis accelerometer (acc) that could potentially be used to detect a footstep (e.g. by detecting movements which are then classified as a step). However, the energy overhead due to active sensors can limit their use in the case of resourceconstrained systems. In the next subsection, we analyse the energy requirements of the overall system, by first considering the accelerometer as an option for step counting, along with a sensor-less solution that is more power efficient.

\section{B. System Energy Requirements with Sensor}

Figure 8 shows the process of counting a step that is divided into four stages:

- Stage 1. MCU off due to a power outage;

- Stage 2. MCU Start-up when power is available (i.e. when a pre-defined threshold $V_{t h}$ is reached);

- Stage 3. Counting a step (i.e. MCU configuration, accelerometer initialization, step detection and data retention in the FRAM);

- Stage 4. MCU in low power mode (LPM), after counting a step.

In order to quantify the energy required during these stages and set the right value of $V_{t h}$, the MCU has been characterized using a constant voltage (i.e. $2.4 \mathrm{~V}$ ) and by using the following algorithm:

1) The system is initially in off mode (stage 1).

2) The system is powered and, after the start-up, the MCU is configured (i.e. frequency and internal peripheral setting) and the on-board accelerometer is initialized.

3) A step is validated (accelerometer sampling plus data processing). 
TABLE II

PARAMETER VALUES OF THE SYSTEM IN EACH STAGE

\begin{tabular}{|c|c|c|c|}
\hline Stage & Tasks & $\begin{array}{c}\text { Current } \\
(\mu \mathrm{A})\end{array}$ & $\begin{array}{c}\text { Time } \\
(\mathrm{ms})\end{array}$ \\
\hline 2 & MCU Start-up & 1000 & 0.9 \\
\hline 3 & MCU+acc & 500 & 13.5 \\
\hline 3 & Validate a step & 300 & 3.6 \\
\hline
\end{tabular}

4) It is retained the state in FRAM and enters in LPM.

Table II shows the current and time needed to successfully complete these stages. In particular, the MCU start-up current is much higher than the other stages, while the accelerometer requires a very long time to be set up.

The values in Table II will be used in the following analysis to evaluate the optimum value for $V_{t h}$ which can be defined as:

$$
V_{t h}=V_{\min }+\frac{1}{C} \int_{0}^{t} I(\tau) d(\tau)
$$

where $V_{\min }$ is the minimum operating voltage of the MCU $(1.8 \mathrm{~V}), C$ is the decoupling capacitance and $I$ is the current consumption. From Table II, Stage 3 has two different subtasks that require different current values. The highest current value refers to $\mathrm{MCU}$ configuration and accelerometer initialization, while the second value refers to the current needed to validate a step. Thus, the Equation 2 can be presented as follows:

$$
V_{t h}=V_{\text {min }}+\frac{1}{C}\left(I_{s u} t_{s u}+I_{a c c} t_{a c c}+I_{v s} t_{v s}\right)
$$

where $I_{s u}$ and $t_{s u}$ are the current and the time needed for the start-up (Stage 2), $I_{a c c}$ and $t_{a c c}$ are the current an time for setting the accelerometer (Stage 3), $I_{v s}$ and $t_{v s}$ are the current and time taken by the MCU to validate a step. Combining the values in Table II and the Equation 3, we obtain a value of $V_{t h}$ equal to $4.01 \mathrm{~V}$, that is higher than the maximum operating voltage for the MCU (3.6V).

For the reason underlined above (i.e. high energy overhead), it is not feasible to use the accelerometer in an intermittentlypowered and resource-constrained system. In the next subsection, we present a solution that allows us to build a more energy efficient step counter, without using an active sensor but which delivers a higher accuracy.

\section{Sensor-less System}

An alternative way to implement a step counter without active sensors is to use the energy harvesting source as an event detection sensor, where the availability of energy is an indicator for a footstep. This approach would reduce the energy required to validate a step, removing the overhead for the accelerometer setting and data processing. Moreover, this will possibly increase the accuracy of the system discarding 'false' steps.

Thus, the MCU needs to be re-characterized by using the following updated algorithm: the system is powered and, after start-up, the MCU is configured. It then validates a step, retains
TABLE III

PARAMETER VALUES OF THE SYSTEM IN EACH STAGE WITHOUT ACCELEROMETER

\begin{tabular}{|c|c|c|c|}
\hline Stage & Tasks & $\begin{array}{c}\text { Current } \\
(\mu \mathrm{A})\end{array}$ & $\begin{array}{c}\text { Time } \\
(\mathrm{ms})\end{array}$ \\
\hline 2 & MCU Start-up & 1000 & 0.9 \\
\hline 3 & Validate a step & 300 & 0.14 \\
\hline
\end{tabular}

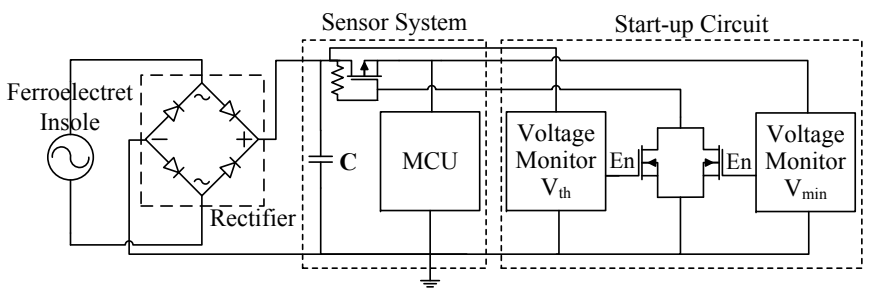

Fig. 9. Intermittently-powered Step Counter circuit diagram.

the state in FRAM and enters in LPM. Table III shows the values for current and time with this algorithm.

The time for counting a step is much smaller than the time needed with the accelerometer. Thus, Equation 3 can be now updated by removing the current and time overhead of the accelerometer:

$$
V_{t h}=V_{\min }+\frac{1}{C}\left(I_{s u} t_{s u}+I_{v s} t_{v s}^{*}\right)
$$

where $t_{v s}^{*}$ is the actual time needed for counting a step. Combining the values from Table III and Equation 4, we obtain a $V_{t h}$ equal to $2.2 \mathrm{~V}$. From Equation 1, the energy required to charge the decoupling capacitance to that voltage is $11.37 \mu \mathrm{J}$. As shown in Table I, the insole is able to charge up to $18.61 \mu \mathrm{J}$ in walking mode, after 3 steps. Therefore, it is possible to implement an event detection sensor based on the energy harvesting source and using the $4.7 \mu \mathrm{F}$ decoupling capacitance.

Figure 9 shows the final design including the ferroelectret insole, the rectifier, the sensor system and an additional startup circuit, which minimizes the quiescent current due to the MCU when its supply voltage is below $V_{\min }$. This circuit guarantees a reliable start by detecting the input voltage and only turning on the supply to the MCU when its input is above $V_{t h}$ and switching it off when the voltage drops below $V_{\text {min }}$. This is enabled by two voltage monitors, which are configured in a MOSFET latch arrangement.

\section{Functional Validation and Comparative EVALUATION}

The presented step counter has been implemented and experimentally validated. We compared the accuracy of our solution against two smartphone applications. To verify system operation, two GPIO pins of the test board were configured to indicate when the system counts and enters the LPM, respectively. Both signals were monitored by a PicoScope at the same time as the voltage across the capacitor and the MCU. 


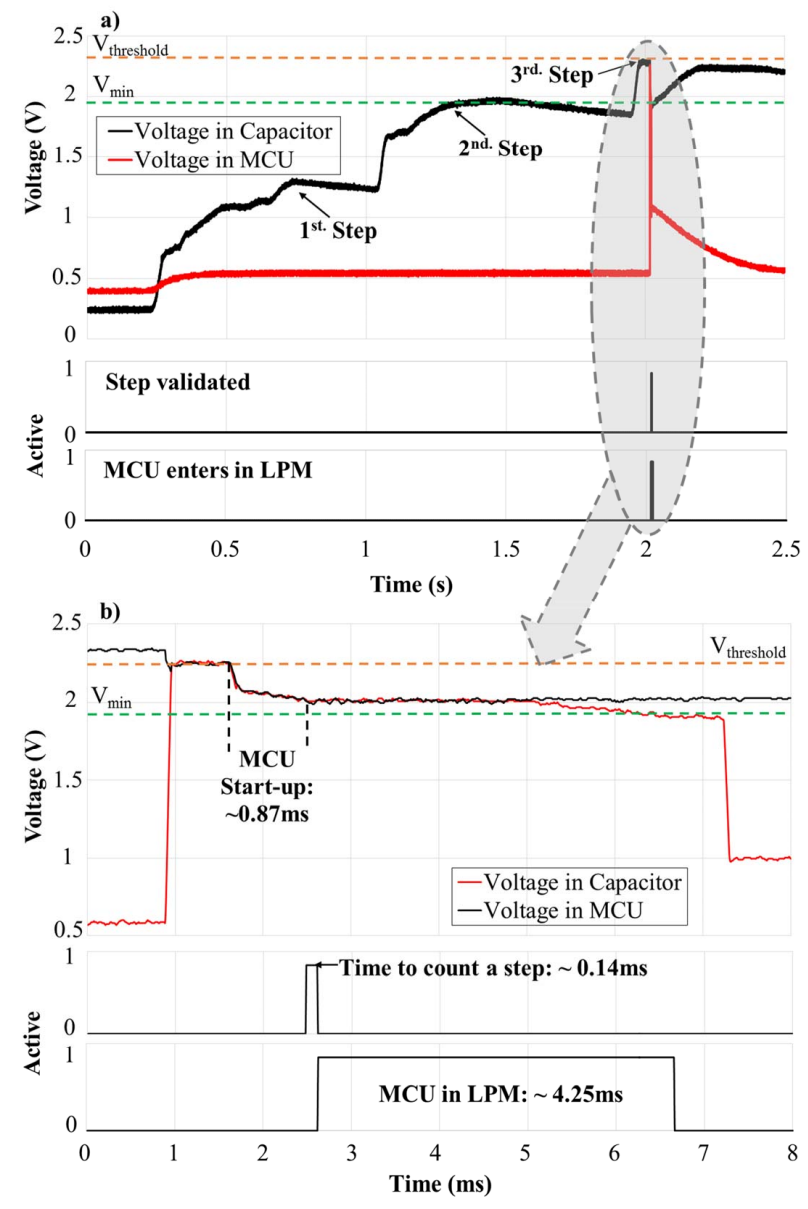

Fig. 10. Operation of the intermittently-powered step counter. a) After three steps the system starts working. b) Detailed description of the process of counting a step.

That information was logged and later plotted. Thus, it was possible to visualize how many steps were taken before the system started working, to detect possible errors. The number of steps is saved in a FRAM variable in order to retain the value between power failures.

As shown in Figure 10a, the system is active when the voltage reaches $V_{t h}$. The sensor node takes into account the initial three steps needed to reach this voltage. Then, the MCU is configured and the step is counted. Once the task is executed, the system enters low power mode before having a power outage. Figure 10b shows a detailed snapshot of the process of counting a step from Figure 10a, including the time needed by the system to start up, configure the MCU and increment the counter. As mentioned in Table III, configuring the MCU and counting a step takes approximately $140 \mu \mathrm{s}$. The MCU remains in LPM until the voltage drops below $V_{\min }$.

Figure 11 shows the system counting three consecutive steps. In walking mode, the average time between two steps is approximately $0.9 \mathrm{~s}$ (worst case). During this interval, the ferroelectret insole does not provide any further energy and the voltage drop, due to the start-up circuit and leakage current, is approximately $0.1 \mathrm{~V}$. This means that the voltage across the

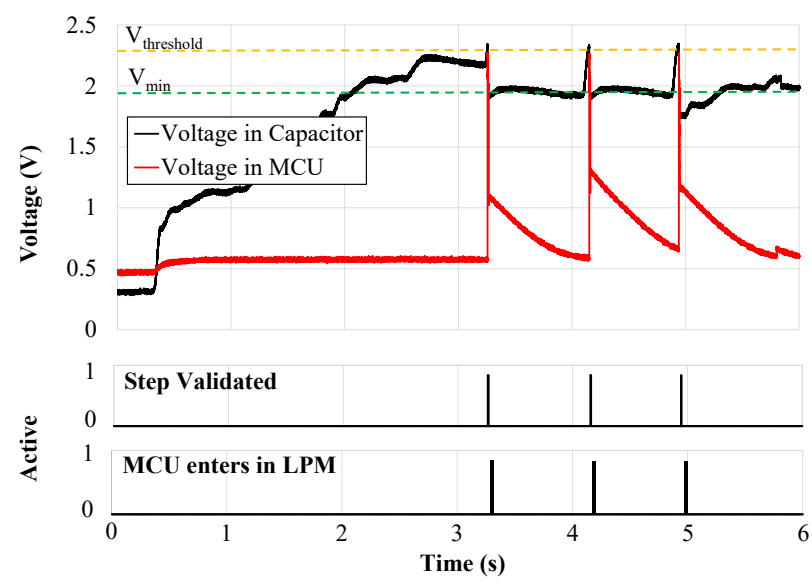

Fig. 11. System counting three consecutive steps.

decoupling capacitance is about $1.7 \mathrm{~V}$, before the next step is taken. Considering that each step in walking mode increases the voltage by $0.8 \mathrm{~V}$, the sensor system is able, after the first three steps, to count each subsequent step.

In order to evaluate the performance of our design, we compared its accuracy against two existing Android ${ }^{\circledR}$ smartphone applications: Pacer [15] and WalkMate [16]. A total of 400 steps were taken per step counter solution (1200 steps in total): 20 steps for 10 attempts in each operating mode. These experiments were performed by a $70-\mathrm{kg}$ person with the ferroelectret insole attached to the shoe and the smartphone placed at his waist. Figure 12 shows the experimental results of our step counter and the two smartphone applications, at each attempt in walking and running mode. The dotted line (in green) represents the real number of steps.

The proposed step counter has an average error of $3.5 \%$ in walking mode, while in running mode the average error is $1 \%$. The maximum error in a single attempt is 2 steps in walking mode (attempts 4 and 9) and 1 step in running mode (attempts 4 and 7). In the case of the first smartphone application (Pacer), it has an average error of $25.5 \%$ and $12.5 \%$ in walking and running modes, respectively. The maximum error in a single attempt is 8 steps (attempt 5) when walking and 4 steps (attempt 4 ) when running. The second application (WalkMate) shows the worst performance in walking mode, having an error rate of $41.5 \%$ with a maximum error of 10 steps in attempts 5, 6 and 10. However, in running mode, this application has a better performance, with an error rate of $4 \%$ and a maximum error of 2 steps in attempts 2 and 5 .

Another test was executed, incrementing the number of steps to 50. Table IV shows the results obtained for each step counter in walking and running mode. Also in this case, our step counter has the lowest error rate in walking mode and it does not present errors in running mode. The WalkMate application has the highest error (44\%) in walking mode, while Pacer had maximum error in running mode $(4 \%)$.

The marginal error in the proposed step counter is due to the adopted solution for compensating the initial number of steps 

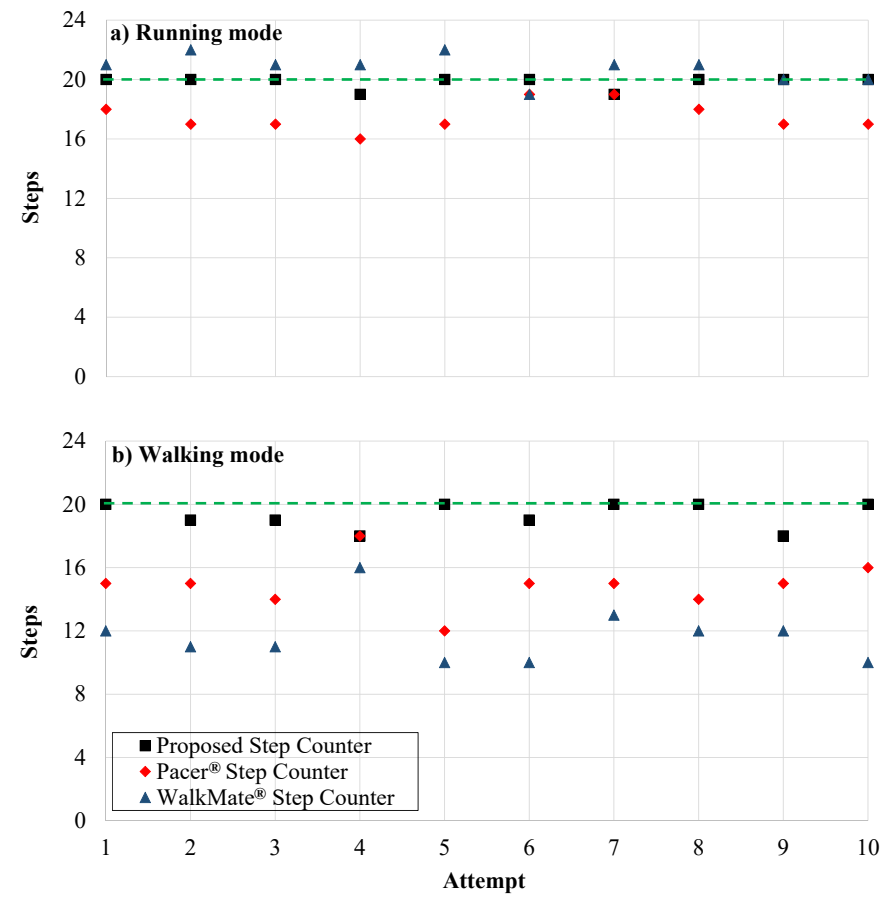

Fig. 12. Performance comparison between the intermittently-powered step counter and two smartphone applications in a) running mode and b) walking mode.

(i.e. when the system is still not active). In a small number of cases, after three steps the voltage across the decoupling capacitance does not reach $V_{t h}$. This mainly happens in walking mode because of the lower energy generated per step. In these cases, the required threshold can be reached after 4 or 5 steps. Despite this, the error rate is less than $4 \%$ in the worst case, which is lower than that of the battery-powered systems.

\section{CONCLUSION}

In this paper, an intermittently-powered energy harvesting step counter for fitness tracking has been proposed. The presented step-counter aims to remove the energy storage element and self-sustains its operation by harvesting energy from footsteps. To achieve this, it uses a ferroelectret insole, which also acts as an event detection sensor. We have characterized the insole to evaluate the amount of energy provided, and analysed of the energy needed by the overall system. The system has been then implemented and experimentally validated. The results show an error of less than $4 \%$ when walking, which is lower than the error in conventional smartphone applications.

\section{ACKNOWLEDGMENT}

This work was supported in part by the Mexican CONACYT. It was also supported by the UK Engineering and Physical Sciences Research Council (EPSRC) Grant EP/L000563/1 (GRACEFUL) and Grant EP/K031910/1 (SPHERE IRC). Experimental data used in this paper can be found at DOI:10.5258/SOTON/405149 (http://doi.org/10.5258/SOTON/405149).
TABLE IV

PERFORMANCE OF THE THREE STEP COUNTERS TESTED WITH 50 STEPS

\begin{tabular}{|c|c|c|c|c|}
\hline System & $\begin{array}{c}\mathrm{N}^{\text {o. Steps }} \\
\text { Walking }\end{array}$ & $\begin{array}{c}\text { Error Rate } \\
(\%)\end{array}$ & $\begin{array}{c}\mathrm{N}^{\text {o. }} \text { Steps } \\
\text { Running }\end{array}$ & $\begin{array}{c}\text { Error Rate } \\
(\%)\end{array}$ \\
\hline $\begin{array}{c}\text { Proposed Step } \\
\text { Counter }\end{array}$ & 49 & 2 & 50 & 0 \\
\hline $\begin{array}{c}\text { Pacer Step } \\
\text { Counter }\end{array}$ & 40 & 20 & 48 & 4 \\
\hline $\begin{array}{c}\text { WalkMate Step } \\
\text { Counter }\end{array}$ & 22 & 44 & 51 & 2 \\
\hline
\end{tabular}

\section{REFERENCES}

[1] J. Williamson, Q. Liu, F. Lu, W. Mohrman, K. Li, R. Dick, and L. Shang, "Data sensing and analysis: Challenges for wearables," in The 20th Asia and South Pacific Design Automation Conference, Jan 2015, pp. 136141.

[2] D. Ferreira, A. K. Dey, and V. Kostakos, Understanding HumanSmartphone Concerns: A Study of Battery Life. Berlin, Heidelberg: Springer Berlin Heidelberg, 2011, pp. 19-33. [Online]. Available: http://dx.doi.org/10.1007/978-3-642-21726-5_2

[3] "Apple Watch. General Battery Information," [Online Document], 2016. Available: http://www.apple.com/watch/battery.html [Accessed: Nov 2016].

[4] "FitBit life expectancy?" [Online Document], 2016. Available: https://www.fitbit.com/ [Accessed: Nov 2016].

[5] J. A. Paradiso and T. Starner, "Energy scavenging for mobile and wireless electronics," IEEE Pervasive Computing, vol. 4, no. 1, pp. 1827, Jan 2005.

[6] H. Huang, G. V. Merrett, and N. M. White, "Humanpowered inertial energy harvesters: the effect of orientation, location and activity on obtainable power," Procedia Engineering, vol. 25, pp. $815-818,2011$. [Online]. Available: http://www.sciencedirect.com/science/article/pii/S1877705811058693

[7] P. Mitcheson, E. Yeatman, G. Rao, A. Holmes, and T. Green, "Energy Harvesting From Human and Machine Motion for Wireless Electronic Devices," Proceedings of the IEEE, vol. 96, no. 9, pp. 1457-1486, sep 2008. [Online]. Available: http://ieeexplore.ieee.org/lpdocs/epic03/wrapper.htm?arnumber=4618735

[8] Z. Luo, D. Zhu, J. Shi, S. Beeby, C. Zhang, P. Proynov, and B. Stark, "Energy harvesting study on single and multilayer ferroelectret foams under compressive force," IEEE Transactions on Dielectrics and Electrical Insulation, vol. 22, no. 3, pp. 1360-1368, Jun 2015. [Online]. Available: http://ieeexplore.ieee.org/lpdocs/epic03/wrapper.htm?arnumber=7116323

[9] J. Hillenbrand and G. M. Sessler, "Piezoelectricity in cellular electret films," IEEE Transactions on Dielectrics and Electrical Insulation, vol. 7, no. 4, pp. 537-542, Aug 2000.

[10] Z. Luo, D. Zhu, and S. P. Beeby, "Multilayer ferroelectret-based energy harvesting insole," Journal of Physics: Conference Series, vol. 660, p. 6, Dec 2015. [Online]. Available: http://stacks.iop.org/1742$6596 / 660 / \mathrm{i}=1 / \mathrm{a}=012118$ ? $\mathrm{key}=$ crossref

[11] D. Balsamo, A. S. Weddell, G. V. Merrett, B. M. Al-hashimi, D. Brunelli, and L. Benini, "Hibernus : Sustaining Computation during Intermittent Supply for Energy-Harvesting Systems," IEEE Embedded Systems Letters, vol. 7, no. 1, pp. 15 - 18, mar 2015.

[12] A. Rodriguez Arreola, D. Balsamo, A. K. Das, A. S. Weddell, D. Brunelli, B. M. Al-Hashimi, and G. V. Merrett, "Approaches to Transient Computing for Energy Harvesting Systems," in Proceedings of the 3rd International Workshop on Energy Harvesting \& Energy Neutral Sensing Systems - ENSsys '15, Seoul, Korea, 2015, pp. 3-8.

[13] “Texas Instruments MSP430FR5739 Datasheet," [Online Document], 2011. Available: http://www.ti.com/lit/ds/symlink/msp430fr5739.pdf.

[14] A. Vazquez, "Novel Piezolectric Transducers for High Voltage Measurements," Ph.D. dissertation, Universitat Politecnica de Catalunya, 2000. [Online]. Available: https://spqr.eecs.umich.edu/papers/ransfordthesis.pdf

[15] "Pacer: Pedometer and Weight Trainer," [Smartphone Application], 2016. Available: http://www.pacer.cc/ [Accessed: Nov 2016].

[16] "Sony WalkMate," [Smartphone Application], 2016. Available: http://www.sonymobile.com/ [Accessed: Nov 2016]. 